\title{
Die Geburt der Medizin aus dem Geiste der Verwundbarkeit
}

$\mathrm{Zu}$ den Binsenweisheiten der philosophischen Anthropologie gehört die Feststellung, dass der Mensch ein zweiseitiges Wesen ist, ein Wesen mit bzw. zwischen Körper und Geist, Leib und Seele, Trieb und Wille, Verstand und Gefühl, Rationalität und Emotionalität um nur einige der zahlreichen Begriffe zu nennen, mit denen dieses fundamentale Spannungsverhältnis beschrieben wird. Je nach disziplinärem Zugang und persönlichem Anliegen werden dabei häufig schon in der Bestimmung des menschlichen Wesens ethische oder politische Positionen akzentuiert. Für den schottischen Moralphilosophen Alasdair MacIntyre etwa ist der Mensch ein »abhängiges rationales Tier « ${ }^{1}$. Darin kommt zunächst die gängige Charakterisierung des Menschen als Vernunftwesen zum Ausdruck - wie sie gerade Philosophen ${ }^{2}$, die ja üblicherweise den Gebrauch ihrer Vernunft besonders schätzen, häufig vornehmen. Aber auch noch eine ganz andere Dimension der menschlichen Existenz ist hier von MacIntyre betont: die körperliche, biologische, tierische Seite. Der Mensch als animal rationale ist eben nicht nur homo sapiens sapiens, sondern gleichzeitig und weiterhin zuallererst ein Naturwesen, ein menschliches Tier. Zuallererst deshalb, weil der natürliche Verlauf eines menschlichen Lebens in körperlichen Zuständen beginnt und endet, die kaum oder gar nicht durch intellektuelle Fähigkeiten oder rationale Leistungen gekennzeichnet sind. Das Menschenkind kommt zur Welt als absolut ausgeliefertes und unselbstständiges Wesen, das allein auf sich gestellt keinen einzigen Tag überlebensfähig ist, das in Gänze dem fordernden Takt seiner körperlichen Grundbedürfnisse $\mathrm{zu}$ folgen gezwungen ist und erst allmählich, nach und nach, zu einem eigenständigen Exemplar seiner Gattung heranreift. Es dauert Monate und Jahre, bis ein neugeborener Mensch sich alleine und ohne Hilfe fortbewegen, ernähren und sprachlich differenziert verständlich machen kann. Und am Ende des Lebens wird der in seiner Lebensmitte von hochgeistigen Fähigkeiten und kognitivrationalem Bewusstsein durchdrungene Kopfmensch Schritt für Schritt wieder zu dem, was er zu Beginn war: ein von der Hilfe

1 Alasdair MacIntyre, Die Anerkennung der Abhängigkeit, Hamburg 2001.

2 Im Folgenden wird zur besseren Lesbarkeit meist nur eine geschlechtliche Form benutzt, es ist jedoch stets sowohl die weibliche als auch die männliche Form gemeint. 
und Pflege anderer abhängiges, mehr und mehr unter der Macht von Primärbedürfnissen und eingeschränkten Körperfunktionen stehendes, unselbstständiges Wesen. Im Tod und in der Geburt ist der Mensch kaum Geist, kaum Verstand und Vernunft, aber völlig Körper.

Abhängig ist der Mensch aber nicht nur an den Polen seiner Existenz, auch während seines Lebens ist er gerade aufgrund seiner biologischen Seite grundsätzlich und dauerhaft der Möglichkeit ausgesetzt, verletzt, gestört und beschädigt zu werden. Neben den vielfältigen Facetten der menschlichen Rationalität wie Sprachkompetenz, Abstraktionsvermögen, Imaginationskraft, der Fähigkeit zu symbolischem Handeln und ähnlichem mehr gehört somit Vulnerabilität zu den Zentralbegriffen einer philosophisch-anthropologischen Bestimmung des Menschen. Dabei ist die grundsätzliche Disposition der Verletzbarkeit allerdings kein Merkmal, das dem Menschen alleine zukommt und ihn von anderen Lebewesen, Pflanzen und Tieren, abhebt. Auch Bäume, Blumen, Vögel, Insekten und Säugetiere sind in ihrer organisch-körperlichen Verfassung prinzipiell bedroht, können verwundet, beschädigt und zerstört werden. Was den Menschen jedoch in besonderer Weise kennzeichnet, ist sein Bewusstsein von und sein Umgang mit dem Umstand seiner Vulnerabilität.

Im Grunde lässt sich die gesamte Zivilisations- und Kulturgeschichte auch verstehen als Prozess der Herausbildung und Verfeinerung von Praktiken und Techniken zur Vermeidung von Beeinträchtigungen der für Schädigungen aller Art anfälligen physischen Konstitution des Menschen. Dabei ist im Verlauf der letzten Jahrhunderte eine Verschiebung bei denjenigen Faktoren erkennbar, von denen lange Zeit die ärgste Bedrohung ausging. In der Frühzeit waren die Menschen noch allerlei widrigen Lebensumständen mehr oder weniger direkt ausgesetzt und behalfen sich mit anfangs nur primitiven Vorkehrungen gegen die beschwerlichen Bedingungen eines wechselvollen Klimas mit heftigen Stürmen und Niederschlägen, großen Temperaturschwankungen zwischen eisiger Kälte und sengender Hitze, wie auch gegen die permanente Bedrohung durch natürliche Feinde, in erster Linie durch wilde Tiere. Nicht zuletzt um diesen externen Gefährdungen zu begegnen und den ungeschützten, nackten Körper vor äußeren Einflüssen zu bewahren und gleichzeitig das Überleben nicht nur zu sichern, sondern auch effizienter und angenehmer zu gestalten, entstanden zivilisatorische Errungenschaften und technische Großleistungen: so etwa die Architektur, der Bau von Sanitär- und Heizungsanlagen, die Per- 
fektionierung von Bekleidung, Landwirtschaft und Tierzucht, die Erfindung und kontinuierliche Fortentwicklung von Maschinen und Fahrzeugen zum Transport und zur Fortbewegung. Somit ist Verwundbarkeit Quelle und Triebfeder zahlreicher Technologien zur Zähmung und Nutzung der wilden Natur sowie zur Kompensation der Unzulänglichkeiten und Gefährdungen der biologischen Ausstattung des Menschen.

Mittlerweile hat sich der Mensch in einem recht komfortablen Leben eingerichtet, angefangen bei bequemen und funktionalen Behausungen mit Zentralheizung und Klimaanlage, bis hin zu einem weitverzweigten System der Lebensmittelproduktion und -versorgung, das die lückenlose Bereitstellung aller erdenklichen Nahrungsmittel weit über das $\mathrm{Maß}$ der Befriedigung der überlebenswichtigen Bedürfnisse hinaus garantiert. Die Lebensumstände (jedenfalls in der westlichen Welt) haben einen Standard erreicht, der einen Großteil der externen Gefahren und Bedrohungen im Normalfall bannt. Der vulnerable Mensch hat sich ein schützendes Gehäuse gebaut und hat Fertigkeiten zur Nutzung seiner Umwelt entwickelt, um seiner prekären Lage zuvorzukommen und sich ein Leben in Wohlstand, Sicherheit und Unversehrtheit zu ermöglichen.

\section{Vulnerabilität und Bioethik}

Doch auch der Mensch des 21. Jahrhunderts ist freilich nicht unverletzlich. Auch er hat einen Körper und ist ein leibliches Wesen, das verwundet und lädiert werden kann. Auch wenn so mancher Transoder Posthumanist diese Achillesferse des homo faber abzuschütteln gewillt ist und die radikale Emanzipation von der Naturhaftigkeit für die nächste Zukunft prognostizieren mag: Von der tatsächlichen Überwindung oder Ablösung des physischen Körpers und seiner biologischen Materialität ist der Mensch noch meilenweit entfernt. Und damit steht auch die vulnerable Seite der menschlichen Existenz vorerst nicht zur Disposition. Im Unterschied zum Frühmenschen, der den ständigen Gefahren sin freier Wildbahn ausgesetzt war, sind aber inzwischen externe Bedrängungen und Hindernisse beherrschbar geworden. Denkt man heute an konkrete Manifestationen menschlicherVulnerabilität, so geraten dementsprechend vermehrt Anfälligkeiten in den Blick, die gegenüber internen Gefährdungsfaktoren bestehen. Den Großteil körperlicher Verletzungen und Beschädigungen haben nicht mehr Raubtiere oder Naturgewalten auf dem Gewissen. In den gesicherten Verhältnissen 
moderner Zivilisationen wächst der Anteil derjenigen Verletzungen, die sich der Mensch selbst bei Unfällen zuzieht. Ob durch Unachtsamkeit, mangelndes Training oder Selbstüberschätzung in Beruf und Freizeit, beim Sport oder im Strassenverkehr: Meist werden dem vulnerablen Menschen also interne, oft selbstverursachte Faktoren zum Verhängnis. Noch deutlicher ist diese Verschiebung im Falle von Erkrankungen, die zum Teil ganz ohne äußere Einflussfaktoren auftreten. Erbkrankheiten, die bereits von Geburt an sim Inneren ihrer Träger angelegt und vorhanden sind, mögen hier als extreme Beispiele gelten. In unseren hochaltrigen Wohlstandsgesellschaften stellen schließlich die körperlichen Ausprägungen des Alterungsprozesses zeitgenössische Paradebeispiele der grundsätzlichen Gebrechlichkeit des Menschen dar.

Während die ausdifferenzierten, weit entwickelten und zum Teil hochtechnisierten Verfahren zur Sicherung von Nahrung, Kleidung und Wohnung Errungenschaften sind, die in unseren Breiten praktisch keine ernsthaften existenziellen Herausforderungen mehr darstellen, ist die Anfälligkeit und potenzielle Verwundbarkeit des Körpers ein dauerhaft bestehendes Problem für alle. Unabhängig von Alter, Geschlecht, vom jeweiligen Wohlstand, dem Bildungsgrad oder ähnlichen persönlichen Faktoren kann jeder Mensch prinzipiell jederzeit verunglücken, sich verletzen oder erkranken. Insofern lässt sich eine weitere Großinstitution menschlicher Kultur auf die Grundbedingung der Vulnerabilität zurückführen: die Medizin. Als Praxis der Hilfe für Menschen in gesundheitlicher Not und Beeinträchtigung hat die Medizin die Abhängigkeit des Menschen im Zentrum ihrer Bestimmung. Alle fundamentalen Zielsetzungen ärztlichen Handelns leiten sich aus dem anthropologischen Umstand der Vulnerabilität ab. Und nicht nur die Ausrichtung der medizinischen Tätigkeit selbst, sondern auch die damit aufs engste verknüpfte ethische Dimension ist ohne Bezug auf die Hinfälligkeit des Menschen kaum verständlich. Für die australischen feministischen Bioethikerinnen und Philosophinnen Wendy Rogers, Catriona Mackenzie und Susan Dodds scheint Vulnerabilität demgemäß »das Herzstück der Bioethik zu sein«³.

3 Wendy Rogers/Catriona Mackenzie/Susan Dodds, Why bioethics needs a concept of vulnerability, in: The International Journal of Feminist Approaches to Bioethics 5 (2012), 11-38, hier 12 (eigene Übersetzung). 


\section{Vulnerabilität und Vertrauen}

Die Medizin lediglich als Praxis der Hilfeleistung und Problemlösung zu bestimmen, bei der Experten unter Zuhilfenahme von fachlichem Wissen und Können Aufträge erledigen, die von Laien an sie herangetragen werden, wäre eine fatale und folgenreiche Verengung ihres Gehalts auf eine bloße Dienstleistung oder pures Handwerk. ${ }^{4}$ Zwar verfügen auch Handwerkerinnen und Dienstleister über Kompetenzen und Fertigkeiten, die ihren Kunden in der Regel fehlen und die diese veranlassen, sich an die Fachleute zu wenden, um von deren Hilfe zu profitieren. Was die Adressaten von Ärztinnen und Ärzten aber von Kundinnen und Konsumenten unterscheidet, ist ihre jeweilige Situation und Rolle. Die Situation ist gekennzeichnet von körperlichen Einschränkungen, Schmerzen und Leidenszuständen, und ihre Rolle ist die von Patienten und Patientinnen. Aus ihren Zuständen erwächst eine Dringlichkeit, die sie in wesentlicher Hinsicht abhebt von kontingenten Konsumwünschen, verzichtbaren Aufträgen an Dienstleister und Handwerkerinnen oder episodischen Zuständen der Kauflust. Verletzte, erkrankte und leidende Menschen benötigen Hilfe, stehen oft in beträchtlichem Maße in ihrer Wahrnehmung, Reflexions- und Kommunikationsfähigkeit unter dem Eindruck ihres Schmerzes und ihrer Beeinträchtigung. Wo ein gesunder Mensch ohne Zeitdruck die verschiedensten Angebote sichten, einschätzen und vergleichen, sich bei anderen Rat und Empfehlungen holen kann, bevor er sich wohlüberlegt und durchkalkuliert schließlich in aller Ruhe zum Kauf oder Auftrag entschließt, macht es die Lage von Patientinnen und Patienten oft gerade aus, dass sie aufgrund ihrer Erkrankung oder Verletzung weder die Nerven noch die Kraft besitzen, die möglichen ärztlichen Hilfsangebote und verschiedenen Behandlungsoptionen eingehend zu prüfen und auszuwählen. In akuten Notfallsituationen ist dies besonders evident, wenn es prinzipiell, d.h. für die direkte Erbringung der nötigen Hilfeleistung nebensächlich ist, welcher Notfallretter oder welche Ärztin erste Hilfe leistet. Oft ist es der betreffenden Person ohnehin gar nicht möglich, darüber zu bestimmen, wer welche medizinischen Schritte vornimmt, weil ihm unfallbedingt das Bewusstsein oder die Klarheit des Verstandes fehlt. Aber auch Menschen, die ärztliche Hilfe benötigen, ohne sich in akuten Notlagen oder einer von starken Schmerzen beeinträchtigten Verfassung zu

\footnotetext{
4 Siehe auch Tobias Eichinger, Die Kehrseite der Selbstbestimmung - Zu Risiken und Nebenwirkungen wunscherfüllender Medizin, in: Selbstbestimmung - zur Analyse eines modernen Projekts, hg. v. Klaus Brücher, Berlin 2015, 256-270.
} 
befinden, sind in einem gewissen $\mathrm{Maß}$ von der Einschätzung, dem Urteilsvermögen und fachlichen Können der behandelnden Ärztin abhängig. Sie sind darauf angewiesen, dieser zu vertrauen.

Gerade der Patient, der ja vom Ausgang einer Behandlung in der Regel abhängig ist wie keine andere Person, muss sichergehen können, dass das ärztliche Handeln eigenen und unumstößlichen Zielen folgt. Dieses Vertrauen ist die unabdingbare Voraussetzung für einen eigentlich ja merkwürdigen oder zumindest bemerkenswerten Umstand: dass sich die Patientin oder der Patient nicht nur im übertragenen Sinne in die Hände eines oft gänzlich fremden Menschen begibt. Vor allem in Fällen, in denen eine Patientin die von ihr aus- und aufgesuchte Ärztin nicht kennt, diese ihr also nicht vertraut ist, ist diese Vertrauensannahme die notwendige Bedingung für die Aufnahme einer Arzt-Patient-Beziehung. Dabei muss sich dieser Vertrauensvorschuss auf den gesamten Berufsstand beziehen, sonst verfehlt er seinen Zweck. Der Patient muss ungeachtet der persönlichen Eigenschaften und Merkmale des einzelnen Arztes allen Vertreterinnen und Vertretern des ärztlichen Standes qua ihrer Zugehörigkeit zu dieser Berufsgruppe in der Überzeugung begegnen können, dass das jeweilige Behandeln zuallererst am eigenen (Patienten-)Wohl ausgerichtet ist. Andernfalls müsste die Patientin eben selbst zuerst die ärztliche Verlässlichkeit prüfen, was keine kleine Hürde darstellt. Gerade Patientinnen und Patienten befinden sich ja typischerweise (und im Wortsinne) in einer vulnerablen Situation, sind körperlich oder seelisch empfindlich eingeschränkt. In der Regel sind sie nur bedingt willens und in der Lage, entsprechende Informationen über das ärztliche Angebot und die Verlässlichkeit der jeweiligen Expertise einzuholen, zwischen verschiedenen Alternativen abzuwägen und zu entscheiden. Für diese Menschen bedeutet das Grundvertrauen in die Ärzteschaft an sich eine enorme Entlastung und kann insofern bereits den ersten Schritt auf dem Weg zur Besserung oder gar Heilung darstellen. Dieser Vertrauensvorschuss ist damit ein Effekt des ärztlichen Hilfsversprechens sowie der herausgehobenen Stellung der Medizin und kann als Beleg der besonderen standesgemäßen Integrität des Arztberufs gelten.

\section{Profession Medizin}

Die besondere Vertrauensforderung in der Medizin hat neben der Situation des Ausgeliefert- und Angewiesenseins auf Patientenseite auch einen wissenschaftstheoretischen oder handlungslogischen 
Grund. So ist Medizin keine Wissenschaft vom Typus der Natur-, Geistes oder Sozialwissenschaften, sie ist vielmehr ihrer Grundstruktur und Aufgabenstellung nach als eine "praktische Wissenschaft" angelegt, "als eine wissenschaftliche Disziplin, die Handlungen nicht nur zum Gegenstand hat, sondern sich selbst in Handlungen realisiert $\mu^{5}$. Und so ist eine der zutreffendsten Bestimmungen von Medizin die einer praktischen Handlungswissenschaft. In diesem Begriff ist enthalten, dass Medizin ihr Ziel nicht im Erkenntnisgewinn etwa im Labor und schon gar nicht am Schreibtisch hat, sondern sich wesentlich in einem Handeln vollzieht. Und entscheidend ist dabei, dass dieses ärztliche Handeln niemals Erfolg garantieren kann. Demgemäß verfolgen Ärztinnen und Ärzte nicht Zwecke, die sie durch ihr Tun auf zweckrationale Weise umsetzen, sondern sie orientieren ihr Handeln an Zielen. Diese Ziele bemühen sie sich zu erreichen, indem sie ihr ganzes Wissen und Können einsetzen. Dies bedeutet, dass ein Arzt der Patientin niemals die erfolgreiche Heilung, die Beseitigung einer Krankheit oder von Schmerzen, oder die Wiedererlangung der Gesundheit garantieren kann. Das einzige, was Ärztinnen ihren Patienten versprechen und einlösen können, ist ihre Absicht und ihr Bemühen, alles in ihrer Macht Stehende für das Erreichen des Behandlungsziels zu tun. Gerade aufgrund dieser prinzipiellen Ergebnisoffenheit ist ärztliche Hilfe besonders auf eine klar bestimmte Zielorientierung angewiesen.

Die moralische Grundhaltung, mit der eine Ärztin ihren Beruf ausübt, ist somit für ihre Tätigkeit nicht nebensächlich oder supererogatorisches Beiwerk, sondern stellt vielmehr einen konstitutiven Wesenszug der Medizin als kollektiver Praxis dar. Typische Dienstleistungen, welche im Sinne eines Handwerks nach Wunsch oder Bestellung von den jeweiligen Spezialisten ausgeführt werden, unterscheiden sich von der ärztlichen Tätigkeit ganz wesentlich darin, dass Dienstleister in ihrem Tun nicht primär eigenen Prinzipien folgen. Zumindest nicht solchen Prinzipien, die eine derart selbstverpflichtende, dabei einheitlich und verbindlich gültige Grundhaltung umfassten, dass man sie als moralisch bezeichnen könnte. Es würde auch dem Sinn und Zweck sowie dem Selbstverständnis des Handwerks- und Dienstleistungssektors direkt widersprechen, sich selbst Richtlinien aufzuerlegen und zum normativen Bestandteil der eigenen Berufsidentität zu machen, die so weit über handwerkliche Regeln der Branche hinausgehen, dass sie den Wünschen der auftraggebenden Person regulierend Grenzen setzen könnten. Ganz

5 Wolfgang Wieland, Diagnose. Überlegungen zur Medizintheorie [1975], Warendorf ${ }^{2} 2004,111 f$. 
im Gegenteil ist doch die möglichst wunschgemäße Ausführung und passgenaue Umsetzung der Vorstellungen und Forderungen des Kunden, die als solche nicht weiter hinterfragt werden, das Kernstück jeder offerierten Leistung einer Handwerkerin und Dienstleisterin. Diese Diskrepanz zwischen Medizin und Handwerk im Blick, wird deutlich, wie weit die ärztliche Praxis von reiner Auftragsarbeit entfernt ist, welche wesentlich gewinnorientiert funktioniert und in unverstellt instrumenteller Weise externen Motiven in Form von Bestellungen und Wünschen folgt. Die Medizin dagegen verfügt über einen spezifischen Moralkodex, der sie zu dem Berufstypus einer Profession macht. ${ }^{6}$ Einer Profession, die in besonderer Weise legitimiert und gleichzeitig limitiert ist.

\section{Vulnerabilität und Moralisierung von Gesundheit}

Vulnerabilität kann als Urgrund der Medizin gelten. Medizinisches Denken und Handeln beschränkt sich jedoch nicht nur auf die Behandlung von eingetretenen Erkrankungen und die Hilfe bei bestehenden Leidenszuständen, sondern folgt heute mehr und mehr auch der Logik von Vorsorge und Prävention. Zwar ist im Verlauf der Menschheitsgeschichte eine Vielzahl äußerer Gefahren und natürlicher Bedrohungen für Leib und Leben durch die enormen Leistungen moderner Zivilisation auf ein beherrschbares Maß gemildert worden. Doch bestehen daneben weiter diverse interne Faktoren, die die Gesundheit beeinträchtigen und stören können. $\mathrm{Zu}$ einem signifikanten Teil wurden solche internen Faktoren gerade durch die Bekämpfung der externen Gefahrenquellen hervorgebracht und noch verstärkt. So führten im Zuge der technisch-wissenschaftlichen Fortschrittsentwicklung des modernen Menschen besonders Effekte der Industrialisierung, Maschinisierung und Automatisierung der Arbeits- und Lebenswelt zu einer beträchtlichen Zunahme gesundheitsgefährdender Begleiterscheinungen. Die allseits ersehnte und unaufhaltsam fortschreitende technikgestützte Entlastung von beschwerlicher körperlicher Arbeit, von anstrengender Nahrungsbeschaffung, von aufwendiger und zeitintensiver Fortbewegung, von Anforderungen, welche nicht nur unangenehm waren, sondern immer auch potenzielle Gefährdungen für den Menschen darstellten, hat ihre unübersehbare Kehrseite in Bewegungsmangel, Umweltgiften, Lärmbelastung sowie Über- und Fehlernährung. Diese

\footnotetext{
${ }^{6}$ Vgl. dazu Friedrich Heubel (Hg.), Professionslogik im Krankenhaus. Heilberufe und die falsche Ökonomisierung, Frankfurt 2015.
} 
oft auch als Zivilisationskrankheiten (bzw. Risikofaktoren für deren Auftreten) bezeichneten Phänomene haben als interne Krankheitsursachen die externen früherer Zeiten weitestgehend abgelöst.

Die im Kranksein spürbare Vulnerabilität, die insofern noch weiter als Pathibilität spezifiziert werden kann, manifestiert sich in unserer Zeit also zu einem großen Teil aufgrund menschengemachter Umstände und Bedingungen. Dementsprechend wird mittlerweile großes Augenmerk auf die Vermeidung solch schädlicher Faktoren sowie einer möglichst gesundheitsfördernden Gestaltung des Lebenswandels und alltäglicher Verhaltensweisen gelegt. Vor allem in den industrialisierten und technisierten Teilen der Welt ist sich der Mensch dieser Dimension seiner gesundheitlichen Anfälligkeit in immer größerem Umfang bewusst und weiß zumindest um die eigene Einfluss- und Gestaltungsmacht.Vulnerabilität muss heute nicht mehr in erster Linie mit der unerbittlichen Unberechenbarkeit snatürlicher Schicksalsschläge - die es freilich weiterhin gibt - in Verbindung gebracht werden, sondern offenbart sich häufig in Bezügen, die als vermeidbar oder doch zumindest verzögerbar gelten. Die Zuschreibung derVerantwortung für die Risiken zu erkranken, hinfällig und damit von medizinischer Hilfe abhängig zu werden, verschiebt sich in diesem Zuge zunehmend auf das Individuum. Jeder und jede ist des eigenen gesundheitlichen Glückes Schmied. Aber auch diese Entwicklung nimmt ambivalente Züge an. Bei aller begrüßenswerten Selbstsorge und verantwortungsbewussten Vernunft zeigen sich die Schattenseiten dieses Paradigmenwechsels, wenn Vorbeugung die Gestalt einer moralischen Pflicht annimmt, mit der dann auch eine latent eingeschriebene Schuldzuweisung einhergeht (sowie ein daraus folgendes stets lauerndes schlechtes Gewissen).

Neben der Moralisierung von Gesundheit, Prävention und gesundheitlicher Eigenverantwortung kann der beschriebene Bewusstseinswandel auch zu bedenklichen Auswirkungen führen, wenn präventives Denken zu einer unangemessenen Obsession des Individuums wird, wenn das Gesundheitsbewusstsein etwa weit über konkrete Anfälligkeiten hinausgeht und sich auf immer mehr alltägliche Aspekte des Lebens erstreckt, wenn jede Tätigkeit unter gesundheitlichen Aspekten geprüft wird, wenn Freizeitgestaltung und soziale Beziehungen dem untergeordnet werden, wenn rund um die Uhr sämtliche verfügbaren Körperwerte gemessen und abgeglichen werden, um sich mittels lückenloser Selbst-Überwachung so fit und gesund wie nur möglich zu machen und zu halten. Zudem kann die Last der individuellen Verantwortung für die eigene Gesundheit zu groß werden und gegenteilige Effekte zeitigen. Wo das Prinzip 
der vorausschauenden Selbstverantwortung in diesem Sinne überzogen wird und den Einzelnen beherrschende und überfordernde Züge annimmt, mag das nicht zuletzt auch an der Charakteristik menschlicherVerletzbarkeit liegen. So kann die konstitutive Tatsache menschlicher Vulnerabilität dazu verführen, es mit der Prävention und Gesundheitssicherung zu ernst $\mathrm{zu}$ nehmen und $\mathrm{zu}$ weit $\mathrm{zu}$ treiben. Da der Mensch dank seiner biologisch-körperlichen Verfassung prinzipiell verletzbar ist und bleibt, selbst wenn er sich bester Gesundheit erfreut, ist er niemals von der Forderung nach präventiven Bemühungen entlastet. Dies fügt sich wiederum bruchlos in die Logik von Prävention, über die der Soziologe Ulrich Bröckling schreibt: "Vorbeugen kann man nie genug und nie früh genug. [...] Wer vorbeugen will, darf niemals aufhören. " ${ }^{7}$ Somit befeuern sich zwei unabschließbare bzw. permanente Phänomene gegenseitig: zum einen die anthropologisch eingeschriebene Vulnerabilität des Menschen, die jede und jeden unausweichlich betrifft, und zum anderen das Projekt des Vorbeugens, das seinem Wesen nach stets im Noch-Nicht operiert und bei dem nie sicher ist, ob oder wann es sein Ziel erreicht hat.

\section{Vulnerabilität und Solidarität}

Eine alternative Form des Umgangs mit der potenziellen Anfälligkeit und tatsächlichen Imperfektibilität mag sich da bieten in einem grundsätzlich anderen Zugang zur Bedingung der eigenen Vulnerabilität und zum Ziel des Gesundseins. So dürfte es durchaus vom eigenen Menschenbild und Selbstverständnis abhängen, welche Rolle Vulnerabilität einnimmt und inwiefern sie als belastend oder gar zu bekämpfender Gegner empfunden wird. Wie der Medizinethiker Giovanni Maio herausstellt, impliziert eine solche alternative Herangehensweise schließlich auch ein Gesundheitsverständnis, das abweicht vom Ideal des fitten, voll funktionsfähigen und leistungsstarken Menschen: "Mit dem Bild vom Menschen als eines grundsätzlich vulnerablen und angewiesenen Wesens würde auch Gesundheit in einem ganz anderen Licht erscheinen. Dann wäre Gesundheit nicht mehr nur die Fähigkeit, alles zu können, sondern vielmehr die Fähigkeit, sich in seinem Angewiesensein zu akzeptieren. $1^{8}$

\footnotetext{
7 Ulrich Bröckling,Vorbeugen ist besser... Zur Soziologie der Prävention, in: Behemoth. A Journal on Civilisation 1 (2008), 38-48, hier 42ff.

8 Giovanni Maio, Mittelpunkt Mensch: Ethik in der Medizin. Ein Lehrbuch, Stuttgart 2012, 385.
} 
Eine derartige Selbstsicht beinhaltet auch die Grundlage, der individualisierenden Tendenz, die eine Moralisierung von Gesundheit und Prävention zu eigen ist, entgegenzuwirken. Da es gerade die vulnerable Grundsituation ist, die allen Menschen gleichermaßen zukommt, ist sie auch dasjenige Moment, das alle verbindet. Und die ohnehin ethisch-anthropologisch grundierte Unternehmung der Medizin wird erkennbar auch als ein Projekt der Solidarität, worauf Rogers, Mackenzie und Dodds in ihrem Plädoyer für eine entsprechende Theoriebildung in der Bioethik abstellen: »Ein Fokus auf Vulnerabilität betont unser gemeinsames Menschsein und bietet eine Grundlage für Solidarität. $"{ }^{9}$ Die Einsicht in die vulnerable Natur des Menschen, die jedem einzelnen inhärent ist, und die Akzeptanz dieses Aspekts vermögen insofern eine der stärksten Quellen bilden für die Herausbildung von echtem menschlichen Gemeinsinn und humaner Verbundenheit (oder für die Erinnerung daran). Dies hat auch MacIntyre im Sinn, wenn er die Abhängigkeit des rationalen Tieres, das wir alle sind, ins Zentrum seiner Anthropologie und Ethik stellt. Er unterstreicht, dass sich Solidarität und Hilfe keinesfalls in bloß einseitigen Beziehungen vollziehen, in denen ein gesunder Mensch einem hilfsbedürftigen Mitmenschen Beistand leistet und dieser die Wohltaten nur empfängt. Die Anerkennung der menschlichen Abhängigkeit umfasst weit mehr als nur das Einsehen einer anthropologischen Unausweichlichkeit, sie vollzieht sich vielmehr in Momenten der Begegnung und des Sich-selbst-im-AnderenErkennens:

"Von Bedeutung ist nicht allein, dass in dieser Gemeinschaft Kinder und Behinderte Gegenstand von Fürsorge und Aufmerksamkeit sind. Entsprechend bedeutsam ist auch, dass jene, die keine Kinder mehr sind, in anderen Kindern wiedererkennen, was sie einst waren, dass jene, die noch nicht die Behinderung des Alters erfahren, in den Alten erkennen, was sie selbst einst sein werden, und dass jene, die nicht krank und verletzt sind, in den Kranken und Verletzten erkennen, was sie selbst oft waren, sein werden und jederzeit sein könnten. «10

- Tobias Eichinger ist Oberassistent am Institut für Biomedizinische Ethik und Medizingeschichte der Universität Zürich. Er beschäftigt sich insbesondere mit der sich wandelnden Identität der Medizin, ethischen Fragen von Enhancement, wunscherfüllender Medizin und Medikalisierung sowie der Darstellung von medizinethischen Problemen im Film.

9 Rogers/Mackenzie/Dodds, Why bioethics needs a concept of vulnerability, $31 \mathrm{f}$.

${ }^{10}$ MacIntyre, Die Anerkennung der Abhängigkeit, 172f. 